\title{
Discovery of the first Ca-bearing molecule in space: CaNC ${ }^{\star}$
}

\author{
J. Cernicharo ${ }^{1}$, L. Velilla-Prieto ${ }^{1,2}$, M. Agúndez ${ }^{1}$, J. R. Pardo ${ }^{1}$, J. P. Fonfría ${ }^{1}$, G. Quintana-Lacaci ${ }^{1}$, C. Cabezas ${ }^{1}$, \\ C. Bermúdez ${ }^{1}$, and M. Guélin ${ }^{3}$ \\ ${ }^{1}$ Grupo de Astrofísica Molecular, Instituto de Física Fundamental (IFF-CSIC), C/Serrano 121, 28006 Madrid, Spain \\ e-mail: jose.cernicharo@csic.es \\ 2 Department of Space, Earth and Environment, Chalmers University of Technology, Onsala Space Observatory, \\ 43992 Onsala, Sweden \\ ${ }^{3}$ Institut de Radioastronomie Millimétrique, 300 rue de la Piscine, 38406 Saint Martin d'Hères, France
}

Received 6 June 2019 / Accepted 14 June 2019

\begin{abstract}
We report on the detection of calcium isocyanide, CaNC, in the carbon-rich evolved star IRC+10216. We derived a column density for this species of $(2 \pm 0.5) \times 10^{11} \mathrm{~cm}^{-2}$. Based on the observed line profiles and the modelling of its emission through the envelope, the molecule has to be produced in the intermediate and outer layers of the circumstellar envelope where other metal-isocyanides have previously been found in this source. The abundance ratio of CaNC relative to $\mathrm{MgNC}$ and $\mathrm{FeCN}$ is $\simeq 1 / 60$ and $\simeq 1$, respectively. We searched for the species $\mathrm{CaF}, \mathrm{CaCl}, \mathrm{CaC}, \mathrm{CaCCH}$, and $\mathrm{CaCH}_{3}$ for which accurate frequency predictions are available. Only upper limits have been obtained for these molecules.
\end{abstract}

Key words. molecular data - line: identification - stars: carbon - circumstellar matter - stars: individual: IRC+10216 astrochemistry

\section{Introduction}

Diatomic metal-bearing molecules were predicted to be produced under thermodynamical chemical equilibrium near the photosphere of carbon-rich evolved stars (Tsuji 1973). Some of these species $(\mathrm{NaCl}, \mathrm{KCl}, \mathrm{AlF}$, and $\mathrm{NaF})$ were detected 30 years ago in the circumstellar envelope (CSE) of the carbon-rich star envelope IRC+10216 by Cernicharo \& Guélin (1987). Shortly after this detection, Guélin et al. (1986) reported on the presence of a new free radical in IRC+10216. Although several silicon- and sulfurbearing candidates were proposed at that time, the line carrier was only identified from laboratory measurements to be magnesium isocyanide (Kawaguchi et al. 1993). The emission of $\mathrm{MgNC}$ was mapped in IRC+10216 with the IRAM Plateau de Bure Interferometer by Guélin et al. (1993). Unlike metal-halogen species that are formed near the stellar photosphere, $\mathrm{MgNC}$ was found to arise from a thin shell $15^{\prime \prime}$ in radius where many reactive species such as the carbon chain radicals $\mathrm{C}_{5} \mathrm{H}, \mathrm{C}_{6} \mathrm{H}, \mathrm{C}_{7} \mathrm{H}$, and $\mathrm{C}_{8} \mathrm{H}$, are also detected (Cernicharo et al. 1986a,b; Guélin et al. 1987; Cernicharo et al. 1987a,b; Cernicharo \& Guélin 1996; Guélin et al. 1997). Just one year after the identification of $\mathrm{MgNC}$, sodium cyanide was also detected in IRC +10216 by Turner et al. (1994). MgNC has been also found towards the more evolved carbon star CRL2688 (Highberger et al. 2003).

Since these early works, several more metal cyanides or isocyanides have been found in CSEs. MgCN, AlNC, SiCN, SiNC, $\mathrm{KCN}, \mathrm{FeCN}$, and $\mathrm{HMgNC}$ have been identified in IRC+10216 after their millimeter-wave rotational spectrum was character-

\footnotetext{
^ This work was based on observations carried out with the IRAM $30 \mathrm{~m}$ telescope. IRAM is supported by INSU/CNRS (France), MPG (Germany) and IGN (Spain).
}

ized in the spectroscopic laboratory (Anderson et al. 1994a; Ziurys et al. 1995, 2002; Guélin et al. 2000, 2004; Pulliam et al. 2010; Zack et al. 2011; Cabezas et al. 2013). Many others have been searched for in the same way without success.

Metals such as $\mathrm{Na}, \mathrm{K}, \mathrm{Ca}, \mathrm{Fe}$, and $\mathrm{Cr}$ and/or their cations are found in the gas phase in IRC+10216 (Mauron \& Huggins 2010), pointing towards a rich metal chemistry in the outer CSE. Diatomic halogen metal-bearing species containing Al, Na, or $\mathrm{K}$ are stable closed-shell molecules that mostly form in the hot part of the envelope, close to the star (Cernicharo \& Guélin 1987). However, the triatomic cyanides containing $\mathrm{Mg}, \mathrm{Si}$, or Fe are open-shell radicals that could react even at low temperature with neutral molecules or atoms and may be formed in the outer envelope. The addition of an hydrogen atom to the radicals $\mathrm{MgNC}$ and $\mathrm{SiCN}$ yields stable closed-shell molecules that may be thought to be abundant in IRC +10216 . HMgNC, the hydrogenated form of the abundant metal isocyanide $\mathrm{MgNC}$, has been found in IRC +10216 by Cabezas et al. (2013) with an abundance ratio $\mathrm{N}(\mathrm{MgNC}) / \mathrm{N}(\mathrm{HMgNC}) \simeq 20$. The millimeter spectra of $\mathrm{HSiCN}$ and $\mathrm{HSiNC}$ have been accurately characterized in the laboratory by Sanz et al. (2002). However, these species have so far not been detected in space. For HFeNC and $\mathrm{HFeCN}$ only ab initio calculations are available, showing that the hydrogenated forms of the cyanade and isocyanide compounds are also open-shell species (Redondo et al. 2016).

The chemistry of these metal-bearing isocyanides could be based on the reaction of the metal cations $\left(\mathrm{Mg}^{+}, \mathrm{Fe}^{+}, \mathrm{K}^{+}, \mathrm{Al}^{+}\right.$, and $\mathrm{Si}^{+}$) with other species formed in the CSE (Petrie 1996; Dunbar $\&$ Petrie 2002). The detection of additional metal-bearing species is therefore necessary to provide additional insights into the reactions leading to the formation of these molecules. 
In this Letter we report on the first Ca-bearing species in space, calcium isocyanide (CaNC), and we perform detailed radiative transfer and chemical models to analyse the origin of the observed emission, and the formation mechanisms of this species.

\section{Observations}

In the course of searches for new molecules we have covered a large portion of the $3,2,1$, and $0.8 \mathrm{~mm}$ spectrum of IRC +10216 with a high sensitivity using the $30 \mathrm{~m}$ IRAM radio telescope. In the $3 \mathrm{~mm}$ window, the data acquired during the last 35 years cover the $80-116 \mathrm{GHz}$ domain with very high sensitivity $(1-3 \mathrm{mK})$. Examples of these data can be found in Agúndez et al. (2008, 2014), Cernicharo \& Guélin (1996), Cernicharo et al. (2007, 2008), and references therein. Most of the $2 \mathrm{~mm}$ data come from the line survey of IRC+10216 carried out by Cernicharo et al. (2000), complemented with additional data obtained during the search for specific molecular species (see, e.g. Guélin et al. 2004; Agúndez et al. 2008, 2012; Fonfría et al. 2006) and for the study of molecular emission variability with time (Cernicharo et al. 2014; Pardo et al. 2018). The sensitivity of the $2 \mathrm{~mm}$ observations varies between 1.5 and $10 \mathrm{mK}$. The $1 \mathrm{~mm}$ and $0.8 \mathrm{~mm}$ data come from observations carried out during the searches quoted above and from a line survey between 290 and $355 \mathrm{GHz}$ carried out using the new EMIR receivers in 2010 and 2011 with a sensitivity of 2-5 mK, depending on the atmospheric transmission. For frequencies above $250 \mathrm{GHz}$ the spectrometers were two autocorrelators with $2 \mathrm{MHz}$ of spectral resolution and $4 \mathrm{GHz}$ of bandwidth. For all other observations the spectral resolution was $1 \mathrm{MHz}$, provided by filter banks or autocorrelators. Some recent observations used the new fast Fourier transform spectrometers, which cover a bandwidth of $2 \times 16 \mathrm{GHz}$ with a spectral resolution of $\sim 200 \mathrm{kHz}$.

The observing mode, in which we wobbled the secondary mirror by $\pm 90^{\prime \prime}$ at a rate of $0.5 \mathrm{~Hz}$, and the dry weather conditions (sky opacity at $225 \mathrm{GHz}$ was below 0.1 in most observations) ensured flat baselines and low system noise temperatures ( $T_{\text {sys }} \simeq 100-400 \mathrm{~K}$ depending on the frequency). This observing method, with the off-position located at $180^{\prime \prime}$ from the star, provides reference data free from emission from all molecular species but CO (see Cernicharo et al. 2015a). The emission of all other molecular species is restricted to a region $\leq 15-20^{\prime \prime}$ from the star (see, e.g. Guélin et al. 1993; Agúndez et al. 2015, 2017; Velilla et al. 2015; Quintana-Lacaci et al. 2016).

The intensity scale, antenna temperature $\left(T_{\mathrm{A}}^{*}\right)$, was corrected for atmospheric absorption using the ATM package (Cernicharo 1985; Pardo et al. 2001). Each frequency setup was observed for $\sim 2 \mathrm{~h}$, with pointing and focus checks in between using strong nearby quasars. Pointing corrections were always within $2-3^{\prime \prime}$. The $30 \mathrm{~m}$ beam sizes are in the ranges $30^{\prime \prime}-21^{\prime \prime}$ at $3 \mathrm{~mm}, 20^{\prime \prime}-17^{\prime \prime}$ at $2 \mathrm{~mm}$, and $12^{\prime \prime}-9^{\prime \prime}$ at $1 \mathrm{~mm}$. The main-beam antenna temperature can be obtained by dividing $T_{\mathrm{A}}^{*}$ by the mainbeam efficiency of the telescope, which is $0.81,0.59$, and 0.35 at 86,230 , and $340 \mathrm{GHz}$, respectively. Calibration uncertainties for data covering such a long observing period have been adopted to be $10 \%, 15 \%, 20 \%$, and $30 \%$ at $3,2,1$, and $0.8 \mathrm{~mm}$, respectively. Additional uncertainties could arise from the line intensity fluctuation with time induced by the variation of the stellar infrared flux, which has been discovered by Cernicharo et al. (2014) for a few molecules and was recently revisited for most molecules present in IRC +10216 by Pardo et al. (2018). All data have been analyzed using the GILDAS package ${ }^{1}$.

\footnotetext{
http://www.iram. fr/IRAMFR/GILDAS
}

\section{Results}

The data revealed several hundreds of spectral lines that could not be assigned to any known molecular species collected in the public CDMS (Müller et al. 2005) and JPL (Pickett et al. 1998) spectral databases. Most of these lines show the characteristic U-shaped or slightly flatted profiles, with line widths of $29 \mathrm{~km} \mathrm{~s}^{-1}$, indicating that they are formed in the external and intermediate layers of the envelope. Among these unknown features a series of doublets were found at $3 \mathrm{~mm}$ in our early observations prior to 2000. These doublets appear in harmonic relation with integer quantum numbers for the rotational angular momentum. However, the sensitivity of the data at $2 \mathrm{~mm}$ was not enough to detect additional doublets above $113 \mathrm{GHz}$. Only when the total set of data at $2 \mathrm{~mm}$ described in the previous section was available were lines up to $N_{\text {up }}=21$ detected. The observed lines are shown in Fig. 1. No lines above $170 \mathrm{GHz}$ were found because the observed lines at 3 and $2 \mathrm{~mm}$ are weak.

The distortion constant of the carrier of the observed lines is 10 times higher than that of $\mathrm{HC}_{3} \mathrm{~N}$, which has a rotational constant of $4549 \mathrm{MHz}$. However, linear triatomic species such as $\mathrm{MgNC}, \mathrm{MgCN}, \mathrm{AlCN}, \mathrm{AlNC}, \mathrm{SiCN}, \mathrm{SiNC}, \mathrm{FeCN}$, and FeNC have distortion constants between 2 and $6 \mathrm{kHz}$ and rotational constants between $\simeq 4-6 \mathrm{GHz}$. (Kawaguchi et al. 1993; Robinson et al. 1997; Walker \& Gerry 1999; Apponi et al. 2000; Flory \& Ziurys 2011; Zack et al. 2011). The carrier of the doublets might therefore be a molecule containing an ionic bond, that is, a metal-bearing species. Moreover, the presence of doublets at constant separation of $18 \mathrm{MHz}$ indicates that the carrier could have a ${ }^{2} \Sigma$ electronic ground state.

From the observed rotational constant the isocyanide CaNC seems to be a good candidate as carrier of the observed lines. This species was implemented in the MADEX code (Cernicharo 2012) from a fit to the rotational lines observed in the laboratory by Steimle et al. (1993) and Scurlock et al. (1994), from which we derive $B=4048.73259(68) \mathrm{MHz}, D=5.001(2) \mathrm{kHz}$, $\gamma=18.082(12) \mathrm{MHz}$ plus some high-order distortion constants. Accurate frequency predictions can be obtained with these constants for all the rotational lines of CaNC within the frequency range of our observations. Figure 1 shows that all the observed doublets correspond to the rotational lines of CaNC. The high dipole moment of the molecule, 6.985 D (Steimle et al. 1992), has certainly helped in its detection.

The observed line intensities of $\mathrm{CaNC}$ are rather low, and at this level of sensitivity, the isotopologues and vibrationally excited states of other molecules could introduce blending and confusion in identifying weak features. Of the ten doublets reported in Fig. 1, several are affected by blending. The $N=$ $9 \rightarrow 8,10 \rightarrow 9,12 \rightarrow 11,14 \rightarrow 13,16 \rightarrow 15,17 \rightarrow 16$, $18 \rightarrow 17$, and $21 \rightarrow 20$ clearly show the two doublets corresponding to the fine structure of each rotational transitions. The worst case corresponds to the $N=13 \rightarrow 12$ transition, for which the high-frequency component of the doublet is fully blended with one of the lines of the doublet of the $N=11 \rightarrow 10$ transition of $\mathrm{C}_{4} \mathrm{H}$ in its $2 v_{7}$ vibrationally excited state. The lowfrequency component of this transition of $\mathrm{CaNC}$ is blended with $\mathrm{C}_{8} \mathrm{H}$. In some cases, one of the components of each doublet is slightly blended left or right by weak features, as indicated in the figure. For example, the $19 \rightarrow 18$ transition (not shown here) is blended in the red side with $\mathrm{SiC}_{2}, \mathrm{HCCN}$, and with an unknown feature. The left side of this CaNC doublet is blended with a feature from $\mathrm{Si}_{2} \mathrm{C}$ (Cernicharo et al. 2015b). Nevertheless, the emission corresponding to $\mathrm{CaNC}$ is clearly visible between these 

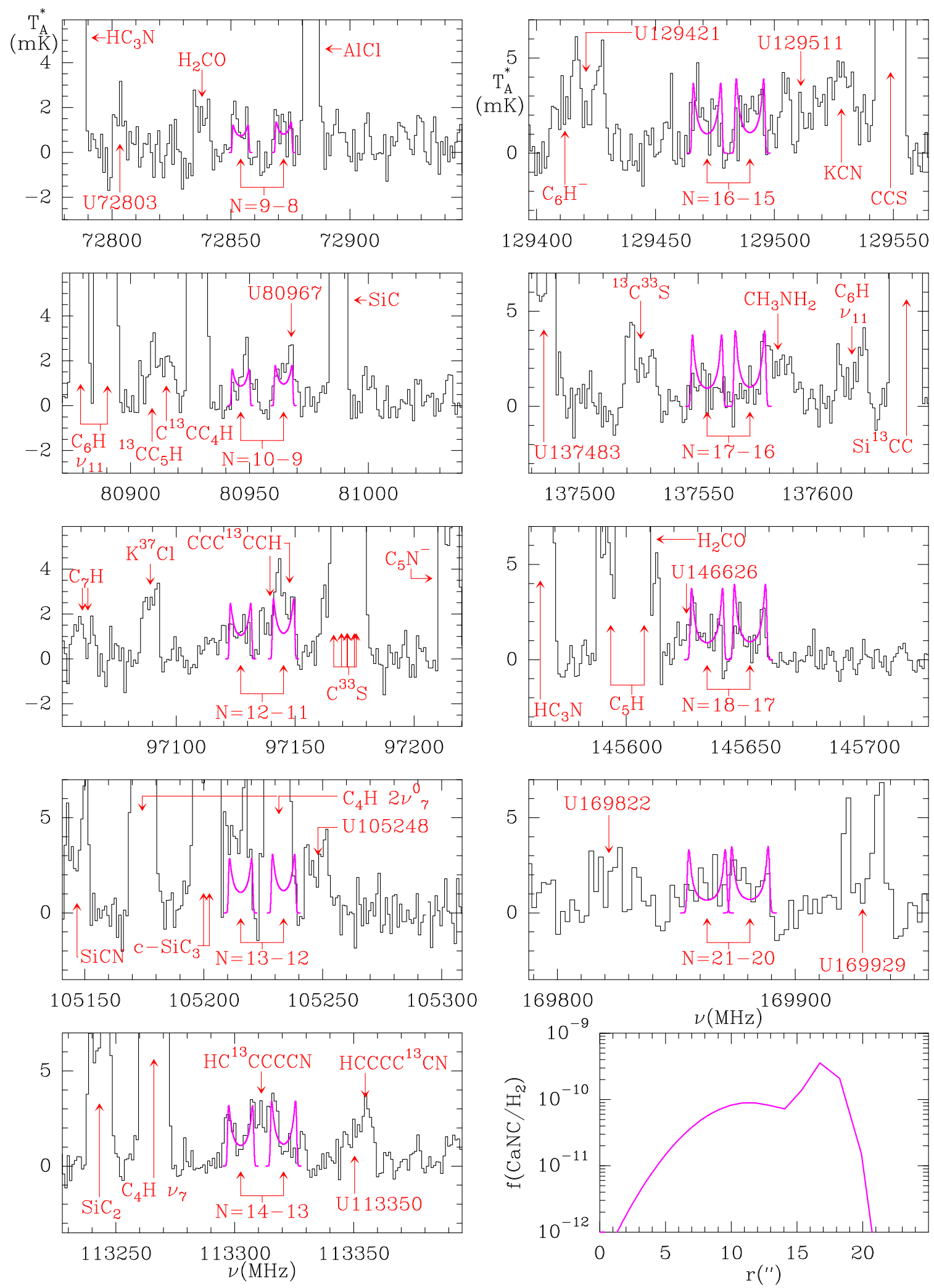

Fig. 1. Results from the radiative transfer models, plotted in magenta, over the IRAM- $30 \mathrm{~m}$ spectrum of IRC+10216, the black histogram. The vertical scale is the antenna temperature in $\mathrm{mK}$, and the horizontal scale is the frequency in MHz. Labels for the visible lines are plotted in red in each panel, where unidentified lines are labelled U lines. The last panel in the bottom-right corner of the figure displays the radial abundance profile in magenta, which we used as input to obtain the synthetic spectra shown in previous panels. The vertical scale of this last panel is the fractional abundance of $\mathrm{CaNC}$ relative to $\mathrm{H}_{2}$, and the horizontal scale is the radial distance to the star in arcseconds.

strong features. As commented previously, the expected intensities of the rotational lines with $N>21$ are very low, and no lines are detected above $170 \mathrm{GHz}$ within the limit of sensitivity of our data. We conclude that the number of observed transitions, with their two-line components, is enough to guarantee the detection of CaNC in IRC+10216. 


\section{Discussion}

We carried out a radiative transfer analysis aiming to derive the radial abundance profile of $\mathrm{CaNC}$ consistent with the emission lines observed. We used the radiative transfer code MADEX (Cernicharo 2012), which computes the population of the levels of a molecule and solves the radiative transfer problem for a spherical circumstellar envelope divided into shells. The populations were calculated using the large velocity gradient (LVG) approximation (Sobolev 1960; Castor 1970) for each shell, starting from equilibrium conditions, and then the radiation field was computed, which is used to derive new populations, following the classical iterative approach until convergence was reached. The implementation of the LVG analysis was based on the formalism of Goldreich \& Kwan (1974). The code then computed the emergent line profile by ray-tracing through the envelope, and by convolving the resulting profile with a Gaussian beam to reproduce the IRAM-30 m main-beam response.

The physical structure of the CSE is essentially an updated revision of the model presented in Agúndez et al. (2012), where we implemented the mass-loss rate of the star and the kinetic temperature radial profile according to the most recent findings (Guélin et al. 2018). There are no theoretical calculations for the collisional rates of CaNC, therefore we used the mass-corrected rates of the similar molecule $\mathrm{MgNC}$ with He (Hernández-Vera et al. 2013), which is valid for the first 41 rotational levels (with $N$ between 0 and 20) in the temperature range [5-100] K. The collisional rates for higher values of $N$ have been extrapolated from the calculated rates for the temperatures of the layers introduced in the modelling.

The results from the radiative transfer models are presented in Fig. 1. Our model is able to reproduce the intensities and line profiles observed with the IRAM-30 m telescope reasonably well. Small discrepancies can be noted for the lines with poor signalto-noise ratios, such as the $N=21-20$ doublet. In addition to these small discrepancies and some blends in particular cases, we conclude that $\mathrm{CaNC}$ emission lines are detected in the millimeter wavelength spectrum of IRC +2016 . According to our models, the $\mathrm{CaNC}$ abundance should be distributed in the intermediate envelope (from 2 to $10^{\prime \prime}$ ) with an average value of $\sim 3 \times 10^{-11}$ (relative to $\mathrm{H}_{2}$ ) and be significantly enhanced in a shell at $\sim 15-20^{\prime \prime}$ up to an average value of $\sim 2 \times 10^{-10}$. The total column density of CaNC is $2 \times 10^{11} \mathrm{~cm}^{-2}$. Using the column density determined by Cabezas et al. (2013) for MgNC, $1.3 \times 10^{13} \mathrm{~cm}^{-2}$, the observed abundance ratio $\mathrm{N}(\mathrm{MgNC}) / \mathrm{N}(\mathrm{CaNC})$ is $\simeq 65$.

The derived column density of $\mathrm{CaNC}$ has to be considered as an upper limit because IR pumping through the low-energy $v_{2}$ bending mode has not been considered in our calculations. Nambu et al. (1997) have performed ab initio calculations for the potential energy surface of $\mathrm{CaNC}$ and found that the bending frequency could be particularly low $\left(\simeq 27 \mathrm{~cm}^{-1}\right)$. Following Agúndez \& Cernicharo (2006) and Agúndez et al. (2017), infrared pumping can significantly increase the population of intermediate and high- $N$ molecular levels with respect to the pure collisional excitation case. This pumping mechanism, which occurs through far-IR photons, can be very efficient indeed, even in the external layers of the envelope where the lines of $\mathrm{CaNC}$ are formed.

The density in the region where CaNC is predicted to be abundantly present, that is, at $15-20^{\prime \prime}$ (or equivalently, $\sim 3 \times 10^{16} \mathrm{~cm}$ considering a distance of 123 pc to the object; Groenewegen et al. 2012), should be close to $\sim 3 \times 10^{4} \mathrm{~cm}^{-3}$, and kinetic temperatures should be close to $40 \mathrm{~K}$. These values have been estimated by using the continuity equation with the mass-loss rate and also the temperature power law derived by Guélin et al. (2018). Given the low densities in this region of the envelope plus the expected values for the critical densities of the transitions here presented $\left(\sim 10^{4-5} \mathrm{~cm}^{-3}\right)$, we cannot rule out uncertainties of a factor two or three in the fractional abundance derived due to radiative pumping effects and approximations considered.

The chemistry of Ca-bearing molecules, including CaNC, in circumstellar envelopes has been discussed by Petrie (2004). This author proposed that $\mathrm{Ca}$-containing cyanides of the type $\mathrm{Ca}\left(\mathrm{C}_{2 n+1} \mathrm{~N}\right)$, with $n=0,1,2$, could be formed in a two-step process initiated by the radiative association of $\mathrm{Ca}^{+}$with long cyanopolyynes followed by the dissociative recombination with electrons of the $\mathrm{Ca}^{+} / \mathrm{NC}_{2 n+1} \mathrm{H}$ complexes, which would fragment into the radicals $\mathrm{Ca}(\mathrm{CN}), \mathrm{Ca}\left(\mathrm{C}_{3} \mathrm{~N}\right)$, and $\mathrm{Ca}\left(\mathrm{C}_{5} \mathrm{~N}\right)$. This mechanism has been proposed to explain the presence of other metal cyanides such as $\mathrm{MgNC}, \mathrm{MgCN}, \mathrm{HMgNC}, \mathrm{NaCN}$, and AlNC in IRC+10216 (Petrie 1996; Dunbar \& Petrie 2002; Millar 2008; Cabezas et al. 2013). The plausibility of this formation mechanism is supported by the high rate coefficients calculated for the reactions of radiative association between the ionized metals $\mathrm{Mg}^{+}, \mathrm{Na}^{+}, \mathrm{Al}^{+}$, and $\mathrm{Ca}^{+}$and cyanopolyynes larger than $\mathrm{HC}_{3} \mathrm{~N}$ (Petrie 1996; Dunbar \& Petrie 2002) and by the observational evidence of the presence of neutral and ionized metals in the outer layers of IRC+10216 (Mauron \& Huggins 2010).

Chemical models have found that $\mathrm{MgNC}, \mathrm{MgCN}$, and $\mathrm{HMgNC}$ can indeed be formed with abundances in agreement with those derived from observations (Millar 2008; Cabezas et al. 2013). To evaluate whether the presence of CaNC in IRC +10216 could similarly be explained by a similar mechanism, we have carried out chemical model calculations based on the recent model by Agúndez et al. (2017) with a chemical network expanded to include metal chemistry as in Cabezas et al. (2013). The chemical network involving $\mathrm{Mg}$, $\mathrm{Na}$, and $\mathrm{Al}$ has been taken from Dunbar \& Petrie (2002), while that involving calcium has been essentially taken from Petrie (2004). The results from our model are shown in Fig. 2.

The observations of Mauron \& Huggins (2010) indicate that gas-phase calcium atoms, which are mostly ionized in the outer shell of IRC +10216 , have an abundance of $6.6 \times 10^{-9}$ relative to hydrogen nuclei. Interestingly, plugging this value as the initial abundance of atomic calcium in the chemical model results in a CaNC abundance of $\sim 10^{-9}$ relative to $\mathrm{H}_{2}$ (see Fig. 1 bottom right panel), somewhat above the value inferred from the observations and radiative transfer calculations. Uncertainties in the calculated abundance come mainly from the reaction rate coefficients, in particular, the branching ratios for the different fragmentations occurring in the dissociative recombination of $\mathrm{Ca}^{+} / \mathrm{NC}_{2 n+1} \mathrm{H}$ complexes. The abundance derived from the observations probably has a large uncertainty due to the unknown effect of IR pumping through its bending mode on the excitation of the rotational levels of CaNC. When we take the different sources of uncertainty into account, the agreement between the calculated and observed abundance is quite satisfactory.

We have searched in our data for the Ca-bearing species for which accurate laboratory data are available: $\mathrm{CaF}$ (Anderson et al. 1994b), CaCl (Möller et al. 1982; Ernst \& Törring 1983; Domaille et al. 1977), CaC (Halfen et al. 2002), $\mathrm{CaCCH}$ (Anderson \& Ziurys 1995), and $\mathrm{CaCH}_{3}$ (Anderson \& Ziurys 1996). These species were implemented in MADEX (Cernicharo 2012) and were searched for in our spectra of IRC +10216 . However, none of then were detected within the sensitivity of the data. We derive $3 \sigma$ upper limits $\simeq 1-3 \times 10^{11} \mathrm{~cm}^{-2}$ for all them. $\mathrm{CaF}, \mathrm{CaCl}$, and probably $\mathrm{CaC}$ might be formed in the innermost regions where the other metal-halogen species 


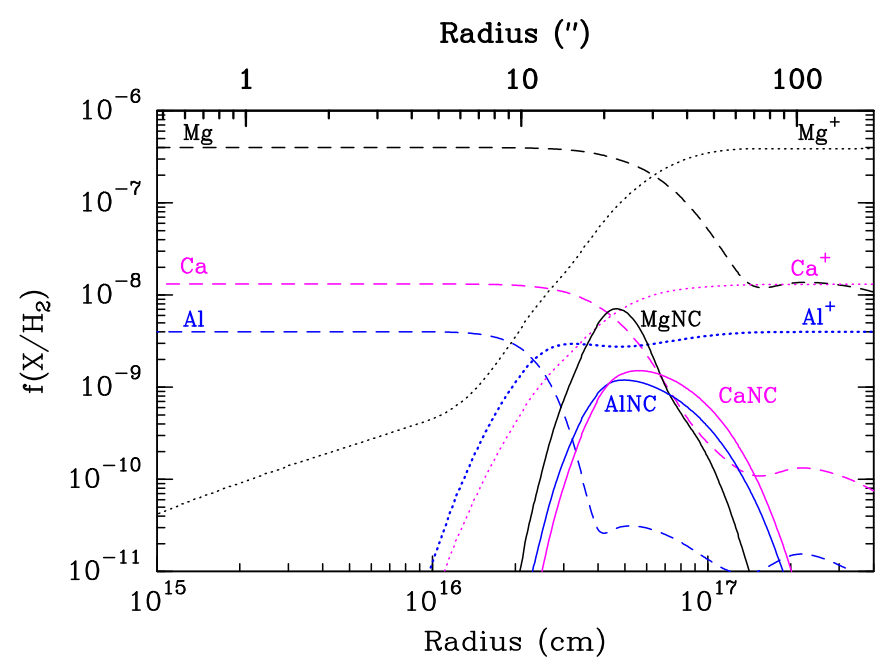

Fig. 2. Abundances, relative to molecular hydrogen, for neutral and ionized $\mathrm{Mg}, \mathrm{Ca}$, and $\mathrm{Al}$, together with those of $\mathrm{MgNC}, \mathrm{AlNC}$, and $\mathrm{CaNC}$ resulting from the model discussed in the text. The lower $X$-axis shows the distance to the star in centimeters, while the upper $X$-axis shows the distance in arcseconds assuming a distance of $123 \mathrm{pc}$ (see text).

have been found (Cernicharo \& Guélin 1987). CaCCH and $\mathrm{CaCH}_{3}$ might be formed in the external layers of the envelope following similar chemical paths to those producing the metal-cyanide species. Except for $\mathrm{MgCCH}$, which has been tentatively detected in IRC +10216 by Agúndez et al. (2014), no detections of other metal-CCH species have been reported so far. There are still many unknown features in our data that could correspond to metal-bearing species, in particular those showing narrow features that are formed in the dust formation and growth zone, 1-20 stellar radii (Cernicharo et al. 2013). While some of these features could correspond to vibrationally excited states of known molecules that are not yet well characterized in the laboratory, many others certainly arise from the building blocks of the seeds of dust grains. A significant laboratory effort has to be performed to find the carriers of these unknown lines.

Acknowledgements. The Spanish authors thank the Ministerio de Ciencia Innovación y Universidades for funding support from the CONSOLIDER-Ingenio program "ASTROMOL" CSD 2009-00038, AYA2012-32032, AYA2016-75066C2-1-P. We also thank the ERC for funding through grant ERC-2013-Syg610256-NANOCOSMOS. MA thanks the Ministerio de Ciencia Innovación y Universidades for the Ramón y Cajal grant RyC-2014-16277. CB thanks the Ministerio de Ciencia Innovación y Universidades for the Juan de la Cierva grant FJCI-2016-27983. LVP acknowledges support from the Swedish Research Council and ERC consolidator grant 614264. We thank our referee, C. Gottlieb, for a critical reading of the manuscript.

\section{References}

Agúndez, M., \& Cernicharo, J. 2006, ApJ, 650, 374

Agúndez, M., Fonfría, J. P., Cernicharo, J., et al. 2008, A\&A, 479, 493 Agúndez, M., Fonfría, J. P., Cernicharo, J., et al. 2012, A\&A, 543, A48 Agúndez, M., Cernicharo, J., \& Guélin, M. 2014, A\&A, 570, 45

Agúndez, M., Cernicharo, J., Quintana-Lacaci, G., et al. 2015, ApJ, 814, 143 Agúndez, M., Cernicharo, J., Quintana-Lacaci, G., et al. 2017, A\&A, 601, A4 Anderson, M. A., \& Ziurys, L. M. 1995, ApJ, 444, L57

Anderson, M. A., \& Ziurys, L. M. 1996, ApJ, 460, L77

Anderson, M. A., Steimle, T. C., \& Ziurys, L. M. 1994a, ApJ, 429, L41

Anderson, M. A., Allen, M. D., \& Ziurys, L. M. 1994b, ApJ, 424, 503

Apponi, A. J., McCarthy, M. C., Gottlieb, C. A., \& Thaddeus, P. 2000, ApJ, 536 L55

Cabezas, C., Cernicharo, J., Alonso, J. L., et al. 2013, ApJ, 775, 133
Castor, J. I. 1970, MNRAS, 149, 111

Cernicharo, J. 1985, Internal IRAM report (Granada: IRAM)

Cernicharo, J. 2012, in ECLA-2011: Proc. Eur. Conf. on Laboratory Astrophysics, EAS Pub. Ser., 58, 251

Cernicharo, J., \& Guélin, M. 1987, A\&A, 183, L10

Cernicharo, J., \& Guélin, M. 1996, A\&A, 309, L27

Cernicharo, J., Kahane, C., Gómez-González, J., \& Guélin, M. 1986a, A\&A, 164, L1

Cernicharo, J., Kahane, C., Gómez-González, J., \& Guélin, M. 1986b, A\&A, 167, L5

Cernicharo, J., Guélin, M., \& Walmsley, C. M. 1987a, A\&A, 172, L5

Cernicharo, J., Guélin, M., Menten, K. M., \& Walmsley, C. M. 1987b, A\&A, 181, L1

Cernicharo, J., Guélin, M., \& Kahane, C. 2000, A\&AS, 142, 181

Cernicharo, J., Guélin, M., Agúndez, M., et al. 2007, A\&A, 467, L37

Cernicharo, J., Guélin, M., Agúndez, M., et al. 2008, ApJ, 688, L83

Cernicharo, J., Daniel, F., Castro-Carrizo, A., et al. 2013, ApJ, 778, L25

Cernicharo, J., Teyssier, D., Quintana-Lacaci, G., et al. 2014, ApJ, 796, L21

Cernicharo, J., Marcelino, N., Agúndez, M., \& Guélin, M. 2015a, A\&A, 575, A91

Cernicharo, J., McCarthy, M. C., Gottlieb, C. A., et al. 2015b, ApJ, 806, L3

Domaille, P. J., Steimle, T. C., \& Harris, D. O. 1977, J. Mol. Spectr., 66, 503

Dunbar, R. C., \& Petrie, S. 2002, ApJ, 564, 792

Ernst, W. E., \& Törring, T. 1983, Phys. Rev. A, 27, 875

Flory, M. A., \& Ziurys, L. M. 2011, J. Chem. Phys., 135, 184303

Fonfría, J. P., Agúndenz, M., Pardo, J. R., et al. 2006, ApJ, 646, L127

Goldreich, P., \& Kwan, J. 1974, ApJ, 189, 441

Groenewegen, M. A. T., Barlow, M. J., Blommaert, J. A. D. L., et al. 2012, A\&A, 543, L8

Guélin, M., Gómez-González, J., Cernicharo, J., \& Kahane, C. 1986, A\&A, 157, L17

Guélin, M., Cernicharo, J., Kahane, C., Gómez-González, J., \& Walmsley, C. M. 1987, A\&A, 175, L5

Guélin, M., Lucas, R., \& Cernicharo, J. 1993, A\&A, 280, L19

Guélin, M., Cernicharo, J., Travers, M. J., et al. 1997, A\&A, 317, L1

Guélin, M., Müller, S., \& Cernicharo, J. 2000, A\&A, 363, L9

Guélin, M., Müller, S., Cernicharo, J., et al. 2004, A\&A, 426, L49

Guélin, M., Patel, N. A., Bremer, M., et al. 2018, A\&A, 610, A4

Halfen, D. T., Apponi, A. J., \& Ziurys, L. M. 2002, ApJ, 577, L67

Hernández-Vera, M., Lique, F., Dumouchel, F., et al. 2013, MNRAS, 432, 468

Highberger, J. L., Thomson, K. J., Young, P. A., et al. 2003, ApJ, 593, 393

Kawaguchi, K., Kagi, E., Hirano, T., et al. 1993, ApJ, 406, L39

Mauron, N., \& Huggins, P. J. 2010, A\&A, 513, A31

Millar, T. J. 2008, Ap\&SS, 313, 223

Möller, K., Schütze-Pahlmann, H. U., Hoeft, J., \& Törring, T. 1982, Chem. Phys., 68, 399

Müller, H. S. P., Schlöder, F., Stutzki, J., \& Winnewisser, G. 2005, J. Mol. Struct., 742,215

Nambu, S., Minamino, S., \& Aoyagi, M. 1997, J. Chem. Phys., 106, 8073

Pardo, J. R., Cernicharo, J., \& Serabyn, E. 2001, IEEE Trans. Antennas Propag., 49, 12

Pardo, J.R., Cernicharo, J., Velilla Prieto, L., et al. 2018, A\&A, 615, L4

Petrie, S. 1996, MNRAS, 282, 807

Petrie, S. 2004, Aust. J. Chem., 57, 67

Pickett, H. M., Poynter, R. L., Cohen, E. A., et al. 1998, J. Quant. Spectrosc. Radiat. Transfer, 60, 883

Pulliam, R. L., Savage, C., Agúndez, M., et al. 2010, ApJ, 725, L181

Quintana-Lacaci, G., Agúndez, M., Cernicharo, J., et al. 2016, A\&A, 592, A51

Redondo, P., Barrientos, C., \& Largo, A. 2016, ApJ, 828, 45

Robinson, J. S., Apponi, A. J., \& Ziurys, L. M. 1997, Chem. Phys. Lett., 278, 1

Sanz, M. E., McCarthy, M. C., \& Thaddeus, P. 2002, ApJ, 577, L71

Scurlock, C. T., Steimle, T. C., Suenram, R. D., et al. 1994, J. Chem. Phys., 100, 3497

Sobolev, V. V. 1960, Moving envelopes of stars (Cambridge: Harvard University Press)

Steimle, T. C., Fletcher, D. A., Jung, K. Y., \& Scurlock, C. T. 1992, J. Chem. Phys., 97, 2909

Steimle, T. C., Saito, S., \& Takano, S. 1993, ApJ, 410, L49

Turner, B. E., Steimle, T. C., \& Meerts, L. 1994, ApJ, 426, L97

Tsuji, T. 1973, A\&A, 23, 411

Velilla, Prieto L., Cernicharo, J., Quintana-Lacaci, G., et al. 2015, ApJ, 805, L13

Walker, K. A., \& Gerry, M. C. L. 1999, Chem. Phys. Lett., 301, 200

Zack, L. N., Halfen, D. T., \& Ziurys, L. M. 2011, ApJ, 733, L36

Ziurys, L. M., Apponi, A. J., Guélin, M., \& Cernicharo, J. 1995, ApJ, 445, L47

Ziurys, L. M., Savage, C., Highberger, J. L., et al. 2002, ApJ, 564, L45 